\title{
WHEAT BRAN EXTRACT MIMICS STATIN LIKE ACTION IN HYPERLIPIDEMIC RABBITS: AN EXPERIMENTAL STUDY
}

\section{RASHI CHADHA*, ASHOK PUROHIT}

\section{Department of Zoology, Jai Narain Vyas University, Jodhpur, Rajasthan, India. Email: rashi24@gmail.com/purohit1411@rediffmail.com}

Received: 16 November 2017, Revised and Accepted: 20 December 2017

\section{ABSTRACT}

Objective: The study was aimed to evaluate the hypolipidemic and antioxidant effect of Triticum aestivum (wheat) bran extraction cholesterol-fed rabbits.

Methods: The induced hyperlipidemic rabbits were treated with $70 \%$ ethanolic extract of wheat bran at a dose of 500 mg/kg body weight/day. The experimental protocol was designed for control, hyperlipidemic, wheat bran extract, and statin-treated groups for 60 days. The estimations for serum lipid profile, lipid peroxidation (LPO), and ferric reducing ability of plasma (FRAP) were done, and histopathological study of thoracic aorta was carried out.

Results: Oral administration of wheat bran extract caused significant reductions in the serum lipid profile by $94.30 \%$ (total cholesterol), $96.73 \%$ (lowdensity lipoprotein cholesterol), and 58.28\% (triglycerides). It significantly decreased the LPO and enhanced the antioxidant capacity (FRAP) activity as compared to the hyperlipidemic rabbits. Histopathology revealed that wheat bran extract treatment prevented the accumulation of cholesterol and regressed atheromatous plaques significantly in the aorta as compared to the hyperlipidemic group, and the results were comparable to that of the standard drug statin.

Conclusion: The study exhibited that wheat bran extract controls atherosclerosis just like statin and can be used as a regular dietary supplement to manage cholesterol levels and prevent cardiovascular problems.

Keywords: Wheat bran, Atherosclerosis, Antioxidant, Hyperlipidemia.

(C) 2018 The Authors. Published by Innovare Academic Sciences Pvt Ltd. This is an open access article under the CC BY license (http://creativecommons. org/licenses/by/4. 0/) DOI: http://dx.doi.org/10.22159/ajpcr.2018.v11i3.23689

\section{INTRODUCTION}

Cardiovascular diseases (CVDs) are one of the leading causes of mortality in both the developed and developing countries. Elevated serum cholesterol level along with generation of reactive oxygen species (ROS) plays a key role in the development of CVD. Hyperlipidemia is a metabolic disorder and its major consequence is atherosclerosis which can be described as a chronic process involving the interplay of inflammation and oxidative stress. Alterations in the serum lipid profile due to raised concentration of total cholesterol (TC), low-density lipoprotein (LDL), very LDL (VLDL), and triglycerides (TGs) with a concomitant lower high-density lipoprotein (HDL) in blood circulation are the hallmark for atherosclerosis [1]. Medicinal plants since time immemorial have been in use for the treatment of various diseases worldwide. Herbs and phytochemicals play a major role in the discovery of new therapeutic agents and have received considerable attention toward the utilization of bio-friendly traditional medicine to meet healthcare needs [2]. Triticum aestivum, commonly called as Wheat, belongs to family Poaceae and is the most widely consumed grain in the world. By weight, the wheat kernel is composed of an outer bran $(14-16 \%$ of the grain), the germ or embryo (2-3\%), and the central endosperm (mainly starch: 81-84\%) [3]. Botanically, the term "wheat bran" refers to the outer layers of the wheat grain comprising the aleurone, pericarp, and seed coat. The bran fractions produced as a by-product of wheat milling are rich in fiber, minerals, Vitamin B6, thiamine, folate, Vitamin E and are a good source of secondary metabolites such as phenolic acids, flavonoids, lignans, and phytosterols [4]. Several studies have shown that high-fat diet increases oxidative stress in plasma and tissues and act as a significant risk factor of CVDs [5]. Oxidation of LDL, recruitment of macrophages, and subsequent uptake of LDL-derived cholesterol by them are the major cellular events contributing to the development of atherosclerotic plaques [6]. A number of medicinal plants have shown their beneficial effect on the CVD by virtue of their lipid-lowering, antianginal, antioxidant, and cardioprotective effects $[7,8]$. Wheat and wheat products contribute significantly toward proteins, vitamins, and minerals when consumed as a major component of diet [9]. Wheat bran is used as a source of dietary fiber and helps in the prevention of colorectal cancer, hemorrhoids, gallbladder disease, hiatal hernia, CVD, obesity, and some gastrointestinal diseases [10]. It is also used for treating constipation, irritable bowel syndrome [11], Type 2 diabetes [12], cholesterol, and high blood pressure [13]. Synthetic medications like statins are used extensively to treat hyperlipidemia but cause several side effects. Hence, the patients are increasingly switching over to herbal products for preventive and therapeutic purposes. The present investigation was carried out with the aim of extrapolating the antiatherosclerotic effect of wheat bran extract in induced hyperlipidemic rabbits.

\section{METHODS}

\section{Extraction of plant material}

Wheat bran was acquired from Morarka organic foods Ltd., Jaipur, and extracted with $70 \%$ ethanol for $36 \mathrm{~h}$ by Soxhlet extraction method. Then, ethanol was separated under reduced pressure to obtain a brownish residue which was resuspended in distilled water and administered orally to the animals.

\section{Model animals}

New Zealand albino male rabbits were used as atherogenic animal models. Weight and age of animals were $1.25-1.50 \mathrm{~kg}$ and $10-12$ months, respectively. The temperature was maintained at $24 \pm 1^{\circ} \mathrm{C}$, humidity at $40-60 \%$, and the daily light cycle was $12 \mathrm{~h}$. The rabbits were kept in in metallic wire gauges with ample space. The regular diet food was supplemented with green leafy seasonal vegetables and water 
ad libitum. The Institutional Animal Ethical Committee approved the protocol (Reg. No. 1646/GO/ERe/S/12/CPCSEA).

\section{EXPERIMENTATION}

\section{Induction of hyperlipidemia}

Rabbits were rendered hyperlipidemic by oral gavage of cholesterol powder at the dose of $500 \mathrm{mg} / \mathrm{kg}$ body weight/day dissolved in $5 \mathrm{ml}$ of coconut oil for 15 days [14].

\section{Experimental design}

The experimental period comprised 60 days and the rabbits were divided into the following groups $(n=5)$ :

1. Group A: Control - placebo treated for 60 days.

2. Group B: Hyperlipidemic control - cholesterol feeding (atherogenic diet + $500 \mathrm{mg}$ cholesterol powder/kg.b.wt./rabbit/day in $5 \mathrm{ml}$ coconut oil) for 60 days.

3. Group C: Wheat bran extract treatment group - cholesterol feeding for 15 days followed by wheat bran extract treatment ( $500 \mathrm{mg} / \mathrm{kg}$. b.wt./rabbit/day) for 45 days.

4. Group D: Statin treatment group - cholesterol feeding for 15 days followed by statin treatment (atorvastatin - $0.25 \mathrm{mg} / \mathrm{kg}$.b.wt./ rabbit/day) for 45 days.

\section{Assessment of serum lipid profile}

At the end of the experiment, all the rabbits were sacrificed under prolonged anesthesia and blood was collected through direct cardiac puncture. Serum was separated by centrifugation and stored at $-20^{\circ} \mathrm{C}$ until analyzed. Serum TC, TG, and HDL-cholesterol were analyzed using Biochemistry Analyzer RX-50, and commercial diagnostic kits (Siemens Healthcare Diagnostics, USA) and LDL-cholesterol, VLDL-C were calculated using Friedewald's formula [15].

\section{Assessment of antioxidant parameters}

Serum lipid peroxidation (LPO) was estimated by measuring the thiobarbituric acid reactive substances and was expressed in terms of malondialdehyde (MDA) content, according to the method of Ohkawa et al. [16].

Serum total antioxidant capacity was measured by the ferric reducing ability of plasma (FRAP) (ferric reducing antioxidant power) assay carried out by according to the modified method of Benzie and Strain [17].

\section{Histopathology of aorta}

The ascending aorta $(2-3 \mathrm{~cm}$ length) was cut at the origin and excised from the heart each animal and kept in $10 \%$ formalin fixative. The processed aorta samples were ultrasectioned (5-6 $\mu \mathrm{m}$ thickness), stained with hematoxylin and eosin (HE), and examined under a light microscope for histopathological observations.

\section{Statistical analysis}

All biochemical estimations were expressed in terms of mean \pm standard error of the mean (SEM). Statistical analysis was carried out by one-way ANOVA followed by Tukey's multiple comparison tests. Data were analyzed using GraphPad Prism-7 software and graphical representations were made using MS Excel 2013.

\section{RESULTS}

\section{Effect on serum lipid profile}

Chronic feeding of cholesterol in rabbits caused significant increase $(p \leq 0.001)$ in the serum lipid profile parameters when compared to the control group. The administration of wheat bran extract (500 mg/kg.b.wt.) caused significant reduction by $94.30 \%$ (TC), 84.07\% (HDL-C), 96.73\% (LDL-C), and 58.28\% (TG) while the standard drug (atorvastatin) treatment group showed reductions by $90.71 \%$, $76.66 \%, 94.03 \%$, and $39.52 \%$ in TC, HDL-C, LDL-C, and TG, respectively, as compared to the hyperlipidemic rabbits (Table 1).

\section{Effect on LPO}

Changes in the lipid peroxides in serum were studied for the effect of wheat bran extract treatment in hyperlipidemic rabbits. High level of MDA formation ( $p \leq 0.001)$ which is the indicator of LPO was seen in the hyperlipidemic group when compared with the control group. The inhibition of LPO was significant $(p \leq 0.01)$ in both the treated groups, wheat bran and standard drug statin, when compared with the hyperlipidemic group (Fig. 1).

\section{FRAP}

To measure the total antioxidant capacity, FRAP value was calculated in terms of $\mu \mathrm{M} / \mathrm{L}$ ascorbic acid equivalents. Induction of hyperlipidemia in rabbits caused highly significant decrease $(\mathrm{P} \leq 0.001)$ in the serum FRAP values. These were restored to near normal values by the treatment of wheat bran extract and statin indicating a positive influence of wheat bran extract on the enzymatic antioxidant defense in hyperlipidemic rabbits (Fig. 2).

\section{Effect on aortic plaque formation - Fig. 3 (i-iv)}

The aorta of animals given T. aestivum bran extract showed almost normal histoarchitecture. However, the aorta of hyperlipidemic rabbits showed fibrofatty plaque with foam cells and spaces within the intima, tunica, and media. Administration of wheat bran extract showed significant protection from aortic lesions. Aortic plaques were regressed significantly and lumen size was also restored near to normal. This effect was comparable to that of the standard drug statin.

\section{DISCUSSION}

New Zealand white male rabbits fed with cholesterol were used for the present study. The formation and distribution of atherosclerotic lesions in the rabbit model resembles atherosclerosis in humans to a great extent [18]. In the present study, administration of cholesterol increased the levels of serum TC, HDL-C, LDL-C, TG, and VLDL-C in rabbits, in accordance with what has been reported previously [19]. The oxidative modification of LDL is predominantly responsible for the initiation and progression of atherogenic changes in the aorta [20]. Atorvastatin has been known to exercise its lipid-lowering effect by competitive inhibition of HMG-CoA reductase, which is the key enzyme for cholesterol biosynthesis. Studies have revealed that terpenoids, steroids, flavonoids, and phenolic compounds are responsible to prevent binding of HMG-CoA to HMG-CoA reductase, either by working individually or synergistically within the plant extract [21]. Our results of wheat bran extract showing similar effect as that of statin in hyperlipidemic rabbits are in agreement with the study by Reddy

Table 1: Serum lipid profile of cholesterol-fed rabbits treated with wheat bran extract

\begin{tabular}{lllll}
\hline Treatment groups & TC (mg/dl) & $\begin{array}{l}\text { HDL cholesterol } \\
\text { (mg/dl) }\end{array}$ & $\begin{array}{l}\text { LDL } \\
\text { cholesterol (mg/dl) }\end{array}$ & $\begin{array}{l}\text { VLDL } \\
\text { cholesterol (mg/dl) }\end{array}$ \\
\hline Group A (intact control) & $53.52 \pm 2.04$ & $22.03 \pm 3.68$ & $19.92 \pm 1.47$ & $12.01 \pm 1.74$ \\
Group B (hyperlipidemic) & $1664.85 \pm 82.39 \mathrm{c}$ & $175.05 \pm 13.28 \mathrm{c}$ & $1369.11 \pm 72.51 \mathrm{c}$ & $53.19 \pm 3.14 \mathrm{c}$ \\
Group C (wheat bran extract) & $94.79 \pm 7.16 \mathrm{a}, \mathrm{g}$ & $27.87 \pm 2.43 \mathrm{~d}, \mathrm{~g}$ & $44.73 \pm 2.97 \mathrm{a}, \mathrm{g}$ & $60.03 \pm 3.71$ \\
Group D (statin) & $154.65 \pm 15.37 \mathrm{~b}, \mathrm{~g}$ & $40.85 \pm 2.23 \mathrm{a}, \mathrm{g}$ & $81.62 \pm 7.47 \mathrm{~b}, \mathrm{~g}$ & $265.97 \pm 15.69 \mathrm{c}$ \\
\hline
\end{tabular}

Values expressed as mean \pm SEM ( $n=5$ ). Group B to D compared with Group A, where $p \leq 0.05=a, p \leq 0.01=b, p \leq 0.001=c$, and non-significant=d. Groups $C$ and $D$ compared with Group B, where $\mathrm{P} \leq 0.05=\mathrm{e}, \mathrm{P} \leq 0.01=\mathrm{f}, \mathrm{P} \leq 0.001=\mathrm{g}$, and non-significant=h. SEM: Standard error of the mean, TC: Total cholesterol, HDL: High-density lipoprotein, LDL: Low-density lipoprotein, VLDL: Very low-density lipoprotein, TGs: Triglycerides 


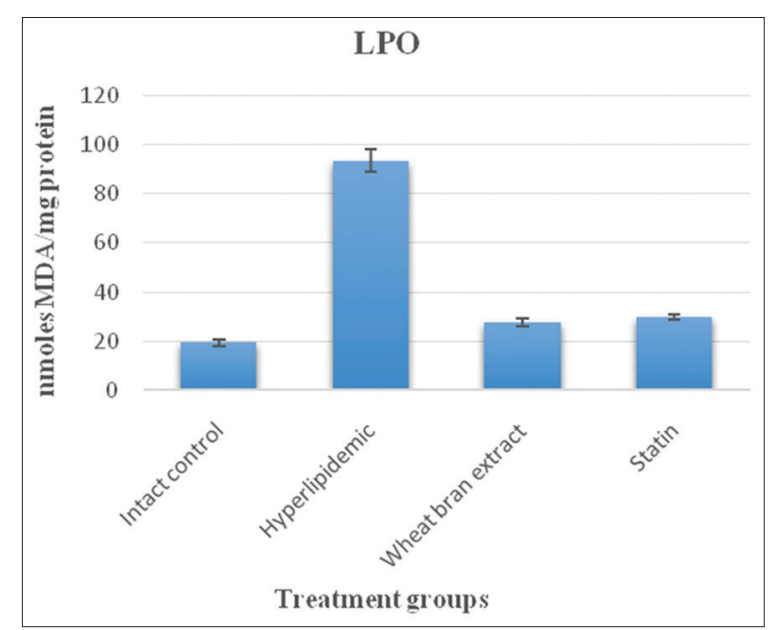

Fig. 1: Effect of Triticum aestivum (wheat) bran extract on lipid peroxidation in nanomoles malondialdehyde/mg protein of various treatment groups (mean of 5 values \pm SEM)

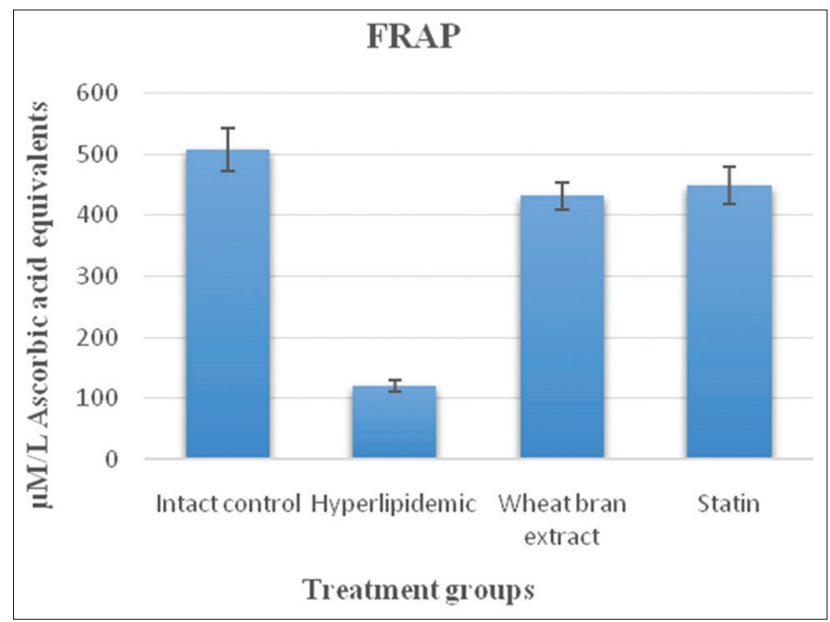

Figure 2: Effect of Triticum aestivum(wheat) bran extract on FRAP values in $\mu \mathrm{M} / \mathrm{L}$ ascorbic acid equivalents of various treatment groups (Mean of 5 values \pm SEM)

et al. who showed in vitro and in vivo HMG-CoA reductase inhibition by Cassia fistula methanolic and ethyl acetate extracts on high-fat-induced hypercholesterolemic rats [22]. Importantly, it was demonstrated that the T. aestivum bran extract reduced plaque formation, cholesterol, and triglyceride levels in rabbits at degrees comparable to that of atorvastatin. The atheromatous changes observed in the aorta of hyperlipidemic and wheat bran-treated rabbits were similar to that of the observations made by Purohit and Ram who noticed regression in atheroma by Prosopis cineraria bark extract treatment in albino rabbits [23]. The inhibition of atherosclerotic plaque formation might be mediated by the improvement of antioxidant status, lipid metabolism, and anti-inflammation response [24].

Carlson et al. suggested that increasing dietary fiber in the diet was associated with a lesser risk of metabolic syndrome [25]. Wheat bran is a by-product of flour milling and is composed of the pericarp and the outermost tissues of the seed which include the aleurone layer. It has an effect on fecal bulking, delays gastric emptying, and accelerates small bowel transit $[26,27]$. The physiological effects of wheat bran can be split into nutritional effects due to the nutrients present in it, mechanical effects mainly on the gastrointestinal tract due to the fiber content and antioxidant effects resulting from the phytonutrients such as phenolic acid and alkylresorcinols present in the bran [28]. The phenolic antioxidants present in wheat bran have been shown to inhibit

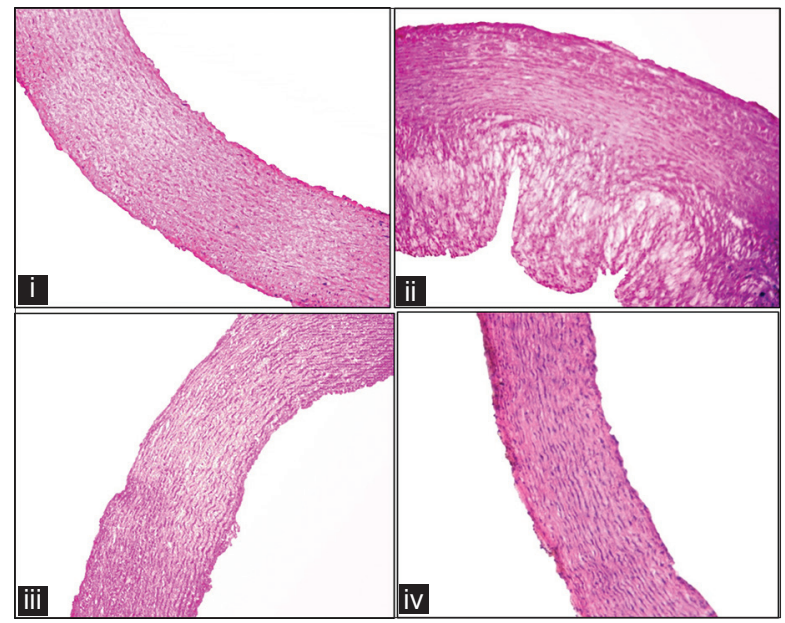

Figure 3: Photomicrographs $(100 \times \mathrm{H}$ and $\mathrm{E})$ of thoracic aorta of treated rabbits - (i) Intact control: Thoracic aorta exhibiting normal histology with aortic wall consisting of tunica adventitia, tunica media, and tunica intima. (ii) Hyperlipidemic: Aortic endothelium with the formation of atherosclerotic plaque which comprises a large number of foam cells and luminal adhesion of mononuclear cells. Intima shows marked increase in thickness

with focal hyalinization in media. (iii) Wheat bran extract:

Intact endothelium with high degree of restoration and mild

proliferation of smooth muscles in tunica media. (iv) Statin:

Thoracic aorta with restored histoarchitecture of the aortic wall

LDL oxidation, possibly by binding with apolipoprotein B [29]. Phytic acid, lignans, and phenolic acids like ferulic acid have been frequently reported as the phytochemicals in wheat bran responsible for health benefits. Consumption of wheat bran fiber in diet is also reported to cause a decrease in plasma cholesterol and TG [30]. In the current investigation, chronic cholesterol feeding in rabbits caused a significant increase in LPO as compared to the control group. LPO refers to the oxidative deterioration of polyunsaturated lipids and MDA is the most abundant product of LPO which indicates tissue damage [30]. Our findings are in agreement with the study by Shallan et al. [31], which demonstrated that wheat bran extract causes significant lipid-lowering and antioxidative activities in hypercholesterolemic rats.

Phytochemicals present in the herbal extracts are known to possess strong free radical and ROS scavenging activity which protects LDL from oxidation [32]. Higher values of FRAP signify higher antioxidant power because the FRAP value is based on reducing ferric ion, where antioxidants act as reducing agent. Significant improvement was observed in the antioxidant status in terms of FRAP value of the serum of rabbits treated with T. aestivum bran extract similar to the observations by Raneva and Shimasaki [33], who studied effect of green tea catechins on atherogenic diet fed mice. Phytosterols are naturally occurring plant sterols that structurally resemble cholesterol. They possess hypolipidemic effect by reducing intestinal cholesterol absorption, thereby enhancing fecal cholesterol excretion and reducing serum LDL cholesterol concentrations [34]. Investigations have shown that intake of fiber also reduces the subsequent ingestion of saturated fat [35]. Wholegrain and bran intakes were associated with reduced CVD-specific mortality in women with diabetes mellitus, suggesting a potential benefit of whole grain intake in reducing mortality and cardiovascular risk in diabetic patients [36]. The antioxidant action of ethanolic extract of wheat bran may be accredited to the presence of bioactive phytocompounds which provide maximum conjugation with reactive free radical species, thus preventing oxidative stress-related ailments like atherosclerosis.

\section{CONCLUSION}

The results of the present study conclude that T. aestivum (wheat) bran extract mimics statin like action in hyperlipidemic rabbits and its oral 
administration could significantly manage serum lipid profile and can be used as a safe and effective hypocholesterolemic and antioxidative agent.

\section{ACKNOWLEDGMENT}

We would like to acknowledge UGC, New Delhi, India, for financial support to Rashi Chadha as SRF (Ref no. 19-06/2011(i) EU-IV) and Head, Department of Zoology, Jai Narain Vyas University, Jodhpur (Rajasthan), for providing all necessary amenities.

\section{AUTHORS CONTRIBUTION}

The complete research work was suggested and designed by Ashok Purohit. All experimentations were carried out by Rashi Chadha. Authors drafted and approved the final manuscript.

\section{CONFLICT OF INTERESTS}

The authors declare no conflict of interests.

\section{REFERENCES}

1. Harrison D, Kathy KG, Hornig B, Drexler H. Role of oxidative stress in atherosclerosis. Am J Cardiol 2003;91:7A-11 A.

2. Subhasree N, Kamella A, Kaliappan I, Agrawal A, Dubey GP. Antidiabetic and antihyperlipidemic activities of a novel polyherbal formulation in high fat diet/streptozotocin induced diabetic rat model. Indian J Pharmacol 2015;47:509-13.

3. Pomeranz Y. Chemical composition of kernel structures. In: Pomeranz Y, editor. Wheat: Chemistry and Technology. Berlin: Springer; 1988. p. 99.

4. Singh B, Sharma HK, Sarkar BC. Optimization of extraction of antioxidants from wheat bran (Triticum spp.) using response surface methodology. J Food Sci Technol 2012;49:294-308

5. de Oliveira Otto MC, Mozaffarian D, Kromhout D, Bertoni AG, Sibley CT, Jacobs DR Jr., et al. Dietary intake of saturated fat by food source and incident cardiovascular disease: The multi-ethnic study of atherosclerosis. Am J Clin Nutr 2012;96:397-404.

6. Glass CK, Witztum JL. Atherosclerosis-the road ahead. Cell 2001:104:503-16.

7. Wang HX, Ng TB. Natural products with hypoglycemic, hypotensive, hypocholesterolemic, antiatherosclerotic and antithrombotic activities. Life Sci 1999;65:2663-77.

8. Dwivedi S. Atherosclerosis revisited. Indian J Cardiol 2004;7:6-12.

9. Shewry PR, Hey SJ. The contribution of wheat to human diet and health. Food Energy Secur 2015;4:178-202.

10. Fardet A. New hypotheses for the health-protective mechanisms of wholegrain cereals: What is beyond fibre? Nutr Res Rev 2010;23:65-134.

11. Kahlon TS, Chow FI, Hoefer JL, Betschart AA. Effect of wheat bran fiber and bran particle size on fat and fiber digestibility and gastrointestinal tract measurements in the rat. Cereal Chem 2001;78:481-4.

12. Liu S, Manson JE, Stampfer MJ, Hu FB, Giovannucci E, Colditz GA, et al. A prospective study of whole-grain intake and risk of Type 2 diabetes mellitus in US women. Am J Public Health 2000;90:1409-15.

13. Kodama T, Shiiba K, Tsuji K. Suppressive effect of Wheat-bran hemicellulose on blood pressure in spontaneously hypertensive Rats. J Jpn Soc Nutr Food Sci 1996;49:101-5.

14. Purohit A, Joshi K, Kotru B. Potential hypolipidemic effects of policosanol (Saccharum officinarum) in hyperlipidemic induced male albino rabbits. World J Pharm Pharm Sci 2014;4:704-14.

15. Friedewald WT, Levy RI, Fredrickson DS. Estimation of the concentration of low-density lipoprotein cholesterol in plasma, without use of the preparative ultracentrifuge. Clin Chem 1972:18:499-502

16. Ohkawa H, Ohishi N, Yagi K. Assay for lipid peroxidation in animal tissues by thiobarbituric acid reaction. Anal Biochem 1979;95:351-8.

17. Benzie I, Strain J. The ferric reducing ability of plasma (FRAP) as a measure of antioxidant power: The FRAP assay. Ana Biochem 1996;239:70-6.

18. Steinberg D. Thematic review series: The pathogenesis of atherosclerosis. An interpretive history of the cholesterol controversy: Part I. J Lipid Res 2004;45:1583-93.

19. Purohit A, Vyas KB. Hypolipidaemic efficacy of capparis decidua fruit and shoot extracts in cholesterol fed rabbits. Indian J Exp Biol 2005;43:863-6.

20. Esterbauer H, Wäg G, Puhl H. Lipid peroxidation and its role in atherosclerosis. Br Med Bull 1993;49:566-76.

21. Wong JP, Wijaya S, Ting KN, Wiart C, Mustafa K, Shipton F, et al. Crude ethanol extract of Pithecellobium ellipticum as a potential lipidlowering treatment for hypercholesterolaemia. Evid Based Complement Alternat Med 2014;2014:492703.

22. Reddy NV, Raj GB, Raju MG, Anarthe SJ. Antihyperlipidemic activity of Cassia fistula bark using high fat diet induced hyperlipidemia. Int J Pharm PharmSci 2015;7:61-4.

23. Purohit A, Ram H. Hypolipidemic and antiatherosclerotic effects of Prosopis cineraria bark extract in experimentally induced hyperlipidemic rabbits. Asian J Pharm Clin Res 2012; 5(3):106-9.

24. Joshi SC, Jain PK, Sharma P. Antiatherosclerotic and lipid-lowering effects of Cinnamomum verum in cholesterol-fed rabbits. Int J Curr Pharm Res 2017;9:75-80.

25. Carlson JJ, Eisenmann JC, Norman GJ, Oritz KA, Young PC. Dietary fibre and nutrient density are inversely associated with metabolic syndrome in US adolescents. J Am Diet Assoc 2011;111:1688-95.

26. McIntyre A, Vincent RM, Perkins AC, Spiller RC. Effect of bran, ispaghula, and inert plastic particles on gastric emptying and small bowel transit in humans: The role of physical factors. Gut 1997;40:223-7.

27. de Vries J, Miller PE, Verbeke K. Effects of cereal fiber on bowel function: A systematic review of intervention trials. World $\mathrm{J}$ Gastroenterol 2015;21:8952-63.

28. Stevenson L, Phillips F, O'Sullivan K, Walton J. Wheat bran: Its composition and benefits to health, a European perspective. Int J Food Sci Nutr 2012;63:1001-13.

29. Yu L, Zhou K, Parry JW. Inhibitory effects of wheat bran extracts on human LDL oxidation and free radicals. LWT Food Sci Tech 2005;38:463-70.

30. Devaraj S, Mathur S, Basu A, Aung HH, Vasu VT, Meyers S, et al. A dose-response study on the effects of purified lycopene supplementation on biomarkers of oxidative stress. J Am Coll Nutr 2008:27:267-73.

31. Shallan M, Fayed S, El M, Gazzar SA. Protective effects of wheat bran and buckwheat hull extracts against hypercholesterolemia in male rats. Int J Adv Res 2014;2:724-36

32. Chumark P, Khunawat P, Sanvarinda Y, Phornchirasilp S, Morales NP. The in vitro and ex vivo antioxidant properties, hypolipidaemic and antiatherosclerotic activities of water extract of Moringa oleifera Lam leaves. J Ethnopharmacol 2008;116:439-46.

33. Raneva VG, Shimasaki H. Green tea catechins decrease lipid peroxidation in plasma and organs of $\mathrm{C} 57 \mathrm{BL} / 6 \mathrm{~J}$ mice fed atherogenic diet. J Oleo Sci 2005;54:641-8.

34. Aziz S, Gohain, K. A study of the hypolipidemic and antioxidant activities of whole plant extracts of Ipomoea aquatica Fork in experimentally induced hyperlipidemia in rabbits. Int J Pharm PharmSci 2016;8:265-9.

35. Jensen MK, Koh-Banerjee P, Hu FB, Franz M, Sampson L, Grønbaek $\mathrm{M}$, et al. Intakes of whole grains, bran, and germ and the risk of coronary heart disease in men. Am J Clin Nutr 2004;80:1492-9.

36. He M, van Dam RM, Rimm E, Hu FB, Qi L. Whole-grain, cereal fiber, bran, and germ intake and the risks of all-cause and cardiovascular disease-specific mortality among women with Type 2 diabetes mellitus. Circulation 2010;121:2162-8. 